\title{
Bone metastases detection by circulating biomarkers: OPG and RANK-L
}

\author{
LAURA MERCATALI $^{1 *}$, TONI IBRAHIM ${ }^{*}{ }^{*}$, EMANUELE SACANNA $^{1}$, EMANUELA FLAMINI ${ }^{1}$, \\ EMANUELA SCARPI ${ }^{1,2}$, DANIELE CALISTRI ${ }^{3}$, MARIANNA RICCI ${ }^{1}$, PATRIZIA SERRA ${ }^{1}$, \\ ROSSANA RICCI ${ }^{1}$, WAINER ZOLI ${ }^{3}$, YIBIN KANG $^{4}$ and DINO AMADORI ${ }^{1}$ \\ ${ }^{1}$ Osteoncology Centre, ${ }^{2}$ Biostatistics and Clinical Trials Unit, ${ }^{3}$ Biosciences Laboratory, \\ Istituto Scientifico Romagnolo per lo Studio e la Cura dei Tumori (IRST), Meldola, Italy; \\ ${ }^{4}$ Department of Molecular Biology, Princeton University, Princeton, NJ, USA
}

Received January 27, 2011; Accepted March 18, 2011

DOI: $10.3892 /$ ijo.2011.1001

\begin{abstract}
Osteoprotegerin (OPG) is a decoy receptor of the receptor activator of nuclear factor- $\kappa \mathrm{B}$ ligand (RANK-L) and plays an important role in the formation of metastatic bone lesions. We evaluated the usefulness of circulating OPG and RANK-L for the detection of bone metastases. We enrolled 143 individuals in the study: 30 healthy donors (HD) and 113 breast cancer patients. Among patients, 49 had no evidence of disease (NEDP), 54 had bone metastases (BMP) at first diagnosis, and 10 had visceral metastases (VMP). Both transcripts were determined in peripheral blood samples using quantitative PCR. Receiver operating characteristic (ROC) curve analysis was used to calculate the diagnostic accuracy of OPG, RANK-L, CEA and CA15-3. OPG and RANK-L median values were significantly lower in BMP (median 0.5, range 0.1-5.7, $\mathrm{p}<0.001$ and median 0.5 , range $0.1-4.5, \mathrm{p}=0.024$, respectively) compared to NEDP (median 1.7, range 0.4-8.9 and median 0.8, range 0.2-3.8, respectively), regardless of the number and type of bone lesions or the presence of visceral metastases. The area under the ROC curve (NEDP vs. BMP) was higher for OPG (82.5, 95\% CI 74.5-90.6) than for RANK-L (69.2, 95\% CI 59.0-79.40). Specificity for OPG was $87.7 \%$ (95\% CI 75.7-94.2) and sensitivity was $74.1 \%$ (95\% CI 60.4-85.0), both values increasing when considered together with CEA and CA15-3. For VMP, OPG and RANK-L were expressed in only one patient. Our results highlight the potentially important role of circulating OPG in the diagnosis of bone metastases. A confirmatory study on a larger case series is ongoing.
\end{abstract}

Correspondence to: Dr Dino Amadori, Osteoncology Centre, Istituto Scientifico Romagnolo per lo Studio e la Cura dei Tumori (IRST), via P. Maroncelli 40, I-47014 Meldola (FC), Italy

E-mail: direzione.scientifica@irst.emr.it

*Contributed equally

Key words: bone metastases, osteoprotegerin, receptor activator of nuclear factor- $\mathrm{\kappa B}$ ligand, breast cancer, diagnosis

\section{Introduction}

The bone represents the third most common site of metastases after the liver and lungs, and breast cancer is the most commonly diagnosed cancer in women in developed countries (1). It has also been reported that over $50 \%$ of breast cancer patients have bone involvement at relapse $(2,3)$. The skeleton is characterized by a dynamic balance between osteoclast-induced bone resorption and osteoblast-stimulated bone formation. Drugs such as Zoledronic acid and Denosumab block this vicious cycle through the inhibition of osteoclasts, not only improving the quality of life of breast cancer patients with bone metastases, but also reducing skeletal-related events and the risk of death (4-6).

The receptor activator of nuclear factor- $\kappa \mathrm{B}$ ligand (RANK-L) binds and activates its receptor RANK on the surface of osteoclasts to stimulate their differentiation and maturation, and at the same time inhibiting osteoclast apoptosis and increasing bone resorption (7). Osteoprotegerin (OPG), expressed by various cell types including osteogenic line cells, acts as a decoy receptor of RANK-L, thereby inhibiting osteoclastogenesis (8). The RANK/RANK-L/OPGaxis governs osteoclastogenesis and bone resorption (9). In particular, RANK is also expressed in tumour cells, while RANK-L, expressed by bone, is thought to be involved in the migration of tumour cells towards bone marrow $(10,11)$. Although the role of these molecules has been investigated in subsets of solid tumours, their relevance with regard to diagnosis of bone metastases has not yet been defined $(7,12)$.

The prevention of bone destruction in metastatic breast cancer not only improves quality of life but also increases survival $(13,14)$. It is therefore vital to diagnose bone metastases before bone destruction occurs. Research is now focusing on the identification of new biomarkers to use alongside, or as an alternative to, conventional instrumental examinations. Several investigators have evaluated new metabolic bone markers by biochemical approaches in urine and blood serum (15-17).

The aim of the present study was to compare the efficacy of circulating tumour markers currently used in clinical practice, CEA and CA15-3, with those involved in the vicious cycle of 
Table I. Marker expressions and pathologic and biologic tumour characteristics in the NED subgroup.

\begin{tabular}{|c|c|c|c|c|c|c|c|c|c|c|c|c|}
\hline & \multirow[b]{2}{*}{$\begin{array}{c}\text { No. of } \\
\text { cases }\end{array}$} & \multirow[b]{2}{*}{$\%$} & \multicolumn{2}{|c|}{ OPG } & \multicolumn{2}{|c|}{ RANKL } & \multicolumn{2}{|c|}{ RANKL/OPG } & \multicolumn{2}{|c|}{ CEA } & \multicolumn{2}{|c|}{ CA15-3 } \\
\hline & & & $\begin{array}{c}\text { Positive } \\
\text { samples } \\
(\%)\end{array}$ & Median & $\begin{array}{c}\text { Positive } \\
\text { samples } \\
(\%)\end{array}$ & Median & $\begin{array}{c}\text { Positive } \\
\text { samples } \\
(\%)\end{array}$ & Median & $\begin{array}{c}\text { Positive } \\
\text { samples } \\
(\%)\end{array}$ & Median & $\begin{array}{c}\text { Positive } \\
\text { samples } \\
(\%)\end{array}$ & Median \\
\hline Overall series & 49 & & & & & & & & & & & \\
\hline \multicolumn{13}{|l|}{ Stage } \\
\hline I & 19 & 38.7 & 15.7 & 1.80 & 31.6 & 0.80 & 15.7 & 0.40 & 7.1 & 1.10 & 6.7 & 18.20 \\
\hline II & 24 & 49.0 & 8.3 & 1.60 & 27.4 & 0.90 & 30.4 & 0.60 & 1.3 & 1.30 & 18.8 & 13.80 \\
\hline III & 6 & 12.3 & 16.6 & 2.00 & 33.3 & 0.60 & 16.7 & 0.30 & 1.2 & 1.20 & 0 & 17.50 \\
\hline IV & 0 & & & & & & & & & & & \\
\hline \multicolumn{13}{|l|}{ Grade } \\
\hline 1 & 3 & 7.2 & 25.0 & 1.70 & 33.3 & 0.70 & 0 & 0.50 & 0 & 0.20 & 0 & 14.50 \\
\hline 2 & 15 & 35.7 & 12.5 & 1.80 & 31.3 & 0.70 & 20.0 & 0.40 & 0 & 1.30 & 0 & 15.30 \\
\hline 3 & 24 & 57.1 & 8.6 & 1.70 & 26.1 & 0.90 & 26.1 & 0.50 & 5.9 & 1.40 & 10.5 & 14.20 \\
\hline Missed & 7 & & & & & & & & & & & \\
\hline \multicolumn{13}{|l|}{ Ki67 (\% positive cells) } \\
\hline$<20$ & 27 & 55.1 & 14.8 & 1.70 & 37.5 & 0.80 & 20.0 & 0.50 & 0 & 1.30 & 5.5 & 13.80 \\
\hline$\geq 20$ & 22 & 44.9 & 9.0 & 1.80 & 21.7 & 0.90 & 26.1 & 0.50 & 5.0 & 1.10 & 17.6 & 17.40 \\
\hline \multicolumn{13}{|l|}{ ER (\% positive cells) } \\
\hline$<10$ & 13 & 34.7 & 0 & 1.90 & 41.6 & 0.80 & 7.7 & 0.30 & 0 & 1.80 & 50.0 & 17.30 \\
\hline$\geq 10$ & 32 & 65.3 & 18.7 & 1.70 & 29.0 & 0.80 & 29.0 & 0.50 & 4.3 & 1.30 & 8.7 & 15.60 \\
\hline Missed & 4 & & & & & & & & & & & \\
\hline \multicolumn{13}{|l|}{ PgR (\% positive cells) } \\
\hline$<10$ & 23 & 46.9 & 13.6 & 1.60 & 23.0 & 0.90 & 10.7 & 0.50 & 5.2 & 1.80 & 50.0 & 18.10 \\
\hline$\geq 10$ & 26 & 53.1 & 11.1 & 1.80 & 38.0 & 0.70 & 22.7 & 0.50 & 0 & 1.10 & 13.3 & 14.90 \\
\hline \multicolumn{13}{|l|}{ HER-2 status } \\
\hline Not amplified $/ 1+^{\mathrm{a}, \mathrm{b}}$ & 30 & 58.3 & 16.7 & 1.50 & 43.3 & 0.60 & 20.0 & 0.50 & 5.2 & 1.30 & 9.7 & 13.80 \\
\hline Amplified $/ 3+^{\mathrm{b}}$ & 18 & 41.7 & 5.7 & 1.90 & 11.1 & 0.80 & 16.6 & 0.50 & 0 & 1.30 & 4.3 & 18.10 \\
\hline Missed & 1 & & & & & & & & & & & \\
\hline
\end{tabular}

${ }^{\mathrm{a}} \mathrm{FISH} ;{ }^{\mathrm{b}} \mathrm{IHC}$.

bone destruction, OPG and RANK-L, to improve the diagnosis of bone metastases.

\section{Materials and methods}

Study design. This was a retrospective observational casecontrol study conducted at the Istituto Scientifico Romagnolo per lo Studio e la Cura dei Tumori (IRST), in Meldola, Italy. The primary objective was to evaluate the diagnostic role of OPG and RANK-L transcripts to detect bone metastases in patients with breast cancer. The secondary objective was to compare these results with those of conventional tumour markers, CEA and CA15-3. Furthermore, all markers were correlated with the biological parameters of the primary tumour. The study was designed to have 2 groups of patients operated on for breast cancer: the first group was composed of patients with no evidence of disease (NEDP); the second consisted of patients with radiologically confirmed bone metastases (BMP). A small series of VM patients was also included as a further negative control. The protocol was reviewed and approved by the Local Ethics Committee and performed according to Good Clinical Practice and the Helsinki declaration. All patients gave their written informed consent to take part in the study.

Patient population. Patients aged $\geq 18$ years, of both sexes, with a histological diagnosis of breast cancer who underwent radical surgery were eligible. Patients were matched for 2 age classes ( $\leq 50$ and $>50$ years). Patients received either no treatment, hormonotherapy or chemotherapy alone or in combination. No patients had active cardiac disease. Exclusion criteria for healthy donors (HD) were the contraceptive pill, hormone replacement therapy and bisphosphonate treatment. The characteristics of primary tumours and metastases are shown in Tables I and II, respectively. Thirty HD (median age 39.5, 
Table II. Marker expressions and pathologic and biologic tumour characteristics in the BM subgroup.

\begin{tabular}{|c|c|c|c|c|c|c|c|c|c|c|c|c|}
\hline & \multirow[b]{2}{*}{$\begin{array}{l}\text { No. of } \\
\text { cases }\end{array}$} & \multirow[b]{2}{*}{$\%$} & \multicolumn{2}{|c|}{ OPG } & \multicolumn{2}{|c|}{ RANKL } & \multicolumn{2}{|c|}{ RANKL/OPG } & \multicolumn{2}{|c|}{ CEA } & \multicolumn{2}{|c|}{ CA15-3 } \\
\hline & & & $\begin{array}{c}\text { Positive } \\
\text { samples } \\
(\%)\end{array}$ & Median & $\begin{array}{c}\text { Positive } \\
\text { samples } \\
(\%)\end{array}$ & Median & $\begin{array}{c}\text { Positive } \\
\text { samples } \\
(\%)\end{array}$ & Median & $\begin{array}{c}\text { Positive } \\
\text { samples } \\
(\%)\end{array}$ & Median & $\begin{array}{c}\text { Positive } \\
\text { samples } \\
(\%)\end{array}$ & Median \\
\hline Overall series & 54 & & & & & & & & & & & \\
\hline \multicolumn{13}{|l|}{ Stage } \\
\hline I & 10 & 20.8 & 70.0 & 0.5 & 66.6 & 0.3 & 40.0 & 0.6 & 22.2 & 19.0 & 44.4 & 89.6 \\
\hline II & 17 & 35.4 & 88.2 & 0.5 & 35.2 & 0.6 & 47.0 & 0.9 & 50.0 & 7.6 & 68.7 & 81.9 \\
\hline III & 9 & 18.8 & 77.7 & 0.7 & 77.7 & 0.4 & 44.4 & 0.6 & 66.6 & 5.4 & 83.3 & 65.1 \\
\hline IV & 12 & 25.0 & 54.5 & 0.6 & 66.6 & 0.4 & 33.3 & 0.7 & 50.0 & 3.7 & 71.4 & 56.9 \\
\hline Missed & 6 & & & & & & & & & & & \\
\hline \multicolumn{13}{|l|}{ Grade } \\
\hline 1 & 1 & 3.1 & 50.0 & 0.2 & 50.0 & 0.7 & 50.0 & 0.6 & 50.0 & 1.9 & 50.0 & 15.7 \\
\hline 2 & 12 & 36.3 & 84.6 & 0.5 & 30.7 & 0.7 & 53.8 & 1.0 & 36.3 & 4.8 & 70.0 & 81.9 \\
\hline 3 & 20 & 60.6 & 72.2 & 0.6 & 76.4 & 0.3 & 38.8 & 0.8 & 53.3 & 9.9 & 66.6 & 32.9 \\
\hline Missed & 21 & & & & & & & & & & & \\
\hline \multicolumn{13}{|l|}{ Ki67 (\% positive cells) } \\
\hline$<20$ & 16 & 41.0 & 68.7 & 0.7 & 73.3 & 0.4 & 40.0 & 0.6 & 71.4 & 4.7 & 90.0 & 12.1 \\
\hline$\geq 20$ & 23 & 59.0 & 71.4 & 0.4 & 63.6 & 0.4 & 50.2 & 1.0 & 78.2 & 5.0 & 61.1 & 48.9 \\
\hline Missed & 15 & & & & & & & & & & & \\
\hline \multicolumn{13}{|l|}{ ER (\% positive cells) } \\
\hline$<10$ & 5 & 10.7 & 68.7 & 0.1 & 60.0 & 0.5 & 60.0 & 2.0 & 66.6 & 6.1 & 100.0 & 161.2 \\
\hline$\geq 10$ & 42 & 89.4 & 71.4 & 0.5 & 59.0 & 0.4 & 45.2 & 0.8 & 48.5 & 4.7 & 60.0 & 64.0 \\
\hline Missed & 7 & & & & & & & & & & & \\
\hline \multicolumn{13}{|l|}{ PgR (\% positive cells) } \\
\hline$<10$ & 11 & 28.2 & 80.0 & 0.6 & 62.5 & 0.4 & 46.6 & 0.8 & 36.0 & 4.4 & 58.3 & 32.4 \\
\hline$\geq 10$ & 28 & 71.9 & 73.0 & 0.5 & 57.1 & 0.5 & 41.3 & 0.8 & 50.0 & 4.6 & 68.1 & 64.8 \\
\hline Missed & 15 & & & & & & & & & & & \\
\hline \multicolumn{13}{|l|}{ HER-2 status } \\
\hline Not amplified/1 $+^{\mathrm{a}, \mathrm{b}}$ & 34 & 77.2 & 73.5 & 4.7 & 66.7 & 67.6 & 35.2 & 0.7 & 48.1 & 0.5 & 69.2 & 0.4 \\
\hline Amplified $/ 3+^{\mathrm{b}}$ & 10 & 22.7 & 80.0 & 2.4 & 50.0 & 36.1 & 60.0 & 1.7 & 30.0 & 0.4 & 33.0 & 0.6 \\
\hline Missed & 10 & & & & & & & & & & & \\
\hline
\end{tabular}

${ }^{\mathrm{a}} \mathrm{FISH} ;{ }^{\mathrm{b}} \mathrm{IHC}$.

range 21-76), and 113 patients with operable breast cancer were enrolled onto the study. Forty-nine patients (median age 61.0, range 30-80) were NEDP after surgery, 54 (median age 63.5, range 34-86) were BMP, and 10 had only visceral metastases (VMP). Regarding the latter group, $50 \%$ were $\mathrm{ER}^{-}$and $\mathrm{PgR}^{+}$, $70 \% \mathrm{Ki}^{+}$, and $71 \%$ HER-2 amplified. Bone metastases were confirmed by scintigraphy, PET scan, CT scan, traditional X-ray or MRI, each patient undergoing at least 2 diagnostic tests, and in 6 patients also by biopsy. The characteristics of BMP are described in Table III.

Cell lines. Human cancer cell lines MCF-7, MDA-MB-231, HT-29 and CAEP (American Type Culture Collection, Rockville, MD, USA) were used as positive controls. Cells were cultured in DMEM/HAM F12 (50/50) supplemented with $10 \%$ fetal calf serum (FCS), 2 mM L-glutamine, $1 \%$ non-essential amino acids and $10 \mathrm{mg} / \mathrm{ml}$ of insulin. Cells were harvested from subconfluent cultures into phosphate-buffered saline (PBS) containing $0.05 \%$ trypsin-0.02\% EDTA (18).

Circulating markers. Markers were determined after surgery and before any systemic treatment in NEDP, and at diagnosis of bone relapse in BMP. The panel of serum markers included CEA, CA15-3, OPG and RANK-L. CEA and CA15-3 assays were routinely performed in the Clinical Pathology Laboratory of Morgagni-Pierantoni Hospital in Forlì, Italy, using AxSYM Chemiluminescent Microparticle Immunoassay and Microplate Enzymatic Immunoassay (Abbott Laboratories, Chicago, IL, 
Table III. Marker expressions and pathologic and biologic tumour characteristics in the BM subgroup.

\begin{tabular}{|c|c|c|c|c|c|c|c|c|c|c|c|c|}
\hline \multirow[b]{2}{*}{ Bone lesions } & \multirow[b]{2}{*}{$\begin{array}{c}\text { No. of } \\
\text { cases }\end{array}$} & \multirow[b]{2}{*}{$\%$} & \multicolumn{2}{|c|}{ OPG } & \multicolumn{2}{|c|}{ RANKL } & \multicolumn{2}{|c|}{ RANKL/OPG } & \multicolumn{2}{|c|}{ CEA } & \multicolumn{2}{|c|}{ CA15-3 } \\
\hline & & & $\begin{array}{c}\text { Positive } \\
\text { samples } \\
(\%)\end{array}$ & Median & $\begin{array}{c}\text { Positive } \\
\text { samples } \\
(\%)\end{array}$ & Median & $\begin{array}{c}\text { Positive } \\
\text { samples } \\
(\%)\end{array}$ & Median & $\begin{array}{c}\text { Positive } \\
\text { samples } \\
(\%)\end{array}$ & Median & $\begin{array}{c}\text { Positive } \\
\text { samples } \\
(\%)\end{array}$ & Median \\
\hline \multicolumn{13}{|l|}{ No. of bone lesions } \\
\hline 1 & 5 & 10.0 & 100.0 & 0.4 & 40.0 & 0.4 & 60.0 & 0.8 & 50.0 & 5.30 & 25.0 & 63.20 \\
\hline $2-4$ & 10 & 20.0 & 60.0 & 0.6 & 80.0 & 0.6 & 10.0 & 0.8 & 14.3 & 4.70 & 42.8 & 71.80 \\
\hline$>4$ & 35 & 70.0 & 25.7 & 0.6 & 54.2 & 0.4 & 48.6 & 0.6 & 60.0 & 6.90 & 76.7 & 134.20 \\
\hline Missed & 4 & & & & & & & & & & & \\
\hline \multicolumn{13}{|l|}{ Type of bone lesions } \\
\hline Lytic & 29 & 58.0 & 84.2 & 0.5 & 50.0 & 0.6 & 52.6 & 0.8 & 58.8 & 5.30 & 50.0 & 64.80 \\
\hline Osteoblastic/mixed & 21 & 42.0 & 73.3 & 0.6 & 68.9 & 0.5 & 37.9 & 0.7 & 36.3 & 4.70 & 78.2 & 69.80 \\
\hline Missed & 4 & & & & & & & & & & & \\
\hline \multicolumn{13}{|l|}{ Visceral metastases } \\
\hline Present & 29 & 65.9 & 73.0 & 0.5 & 66.6 & 0.7 & 50.0 & 0.7 & 50.0 & 3.80 & 62.5 & 46.80 \\
\hline Absent & 15 & 34.1 & 57.6 & 0.6 & 58.3 & 0.6 & 43.7 & 0.7 & 33.3 & 7.40 & 57.1 & 76.00 \\
\hline Missed & 10 & & & & & & & & & & & \\
\hline
\end{tabular}

USA), respectively. The limits of normality were $5 \mathrm{ng} / \mathrm{ml}$ for $\mathrm{CEA}$ and $33 \mathrm{U} / \mathrm{ml}$ for CA15-3. For OPG and RANK-L determinations, peripheral blood samples $(2.5 \mathrm{ml})$ were collected in Paxgene tubes (Becton-Dickinson, Franklin Lakes, NJ, USA) via peripheral vein puncture and the first $5 \mathrm{ml}$ were discarded to avoid possible contamination by epidermal cells.

Blood RNA was extracted by PAX-Gene blood RNA kit (PreAnalytix-Qiagen, Hilden, Germany) in HD and patients, while RNA isolation from cell lines was performed with RNeasy Mini Kit (Qiagen) according to the manufacturer's instructions. RNA was treated with DNAse I (Qiagen) and 500 ng of RNA were reverse-transcribed using the iScript cDNA Synthesis Kit (Bio-Rad, Hercules, CA, USA). The final mixture was incubated at $25^{\circ} \mathrm{C}$ for $5 \mathrm{~min}$, at $42^{\circ} \mathrm{C}$ for $20 \mathrm{~min}$, at $47^{\circ} \mathrm{C}$ for $20 \mathrm{~min}$, at $50^{\circ} \mathrm{C}$ for $15 \mathrm{~min}$ and $5 \mathrm{~min}$ at $85^{\circ} \mathrm{C}$.

Real-time PCR was performed using the MyiQ Single Color Real-Time PCR Detection System (Bio-Rad) and SYBR Green I dye chemistry. The stably expressed endogenous $\beta_{2}$-microglobulin, $\beta$-actin and HPRT genes were amplified and used as reference genes. Primers were designed by Beacon Designer Software (Premier Biosoft International, Palo Alto, CA, USA). Primer sequences are reported in Table IV. After reverse transcription reactions, amplification was performed in a final volume of $25 \mu \mathrm{l}$ containing $0.2 \mu \mathrm{M}$ of primers for housekeeping genes and $0.4 \mu \mathrm{M}$ of primers for OPG and RANK-L, 2X SYBR Green Supermix (Bio-Rad) and $5 \mu \mathrm{l}$ of cDNA diluted 1:2.5. The reaction mixtures were all subjected to $40 \mathrm{PCR}$ cycles at $95^{\circ} \mathrm{C}$ for $90 \mathrm{sec}$, and then to 40 cycles at $95^{\circ} \mathrm{C}$ for $15 \mathrm{sec}$ and $60^{\circ} \mathrm{C}$ for $30 \mathrm{sec}$ for housekeeping genes, and to 40 cycles at $95^{\circ} \mathrm{C}$ for $15 \mathrm{sec}$ and $62^{\circ} \mathrm{C}$ for $45 \mathrm{sec}$ for OPG and RANK-L.

The efficiency of RT-PCR was evaluated on a standard curve of HT-29 cell lines for housekeeping genes and MCF-7 cell lines for OPG. All RT-PCR experiments were run in triplicate. The amount of transcripts was normalized to the
Table IV. Sequence of primers.

\begin{tabular}{lll}
\hline Gene & $\begin{array}{c}\text { Forward/ } \\
\text { Reverse }\end{array}$ & \multicolumn{1}{c}{ Sequence } \\
\hline$\beta_{2}$-micro- & Forward & 5'-CGCTACTCTCTCTTTCTGGC-3' \\
globulin & Reverse & 5'-AGACACATAGCAATTCAGGAAAT-3' \\
$\beta$-actin & Forward & 5'-CGCCGCCAGCTCACCATG-3' \\
& Reverse & 5'-CACGATGGAGGGGAAGACGG-3' \\
HPRT & Forward & 5'-AGACTTTGCTTTCCTTGGTCAGG-3' \\
& Reverse & 5'-GTCTGGCTTATATCCAACACTTCG-3' \\
OPG & Forward & 5'-TGTCTTTGGTCTCCTGCTAAC-3' \\
& Reverse & 5'-AACCTGAAGAATGCCTCCTC-3' \\
RANK-L & Forward & 5'-ATCACAGCACATCAGAGCAGAG-3' \\
& Reverse & 5'-GGACAGACTCACTTTATGGGAACC-3'
\end{tabular}

endogenous reference genes and expressed as N-fold mRNA levels relative to a calibrator using Gene Expression Macrosoftware (version 1.1) (Bio-Rad) using an optimized comparative threshold cycle $(\mathrm{Ct})$ value method $(\Delta \Delta \mathrm{Ct})$. The calibrator used was an arbitrarily selected HD who was analyzed in all the experiments. The intra-assay coefficient of variation $(\mathrm{CV})$ was $<1.5 \%$ and inter-assay CV was always $<15 \%$. When 2 out of 3 replicates did not emit any fluorescence, i.e., there was no amplification production, a value of 0.1 was attributed to the sample.

Statistical analysis. Descriptive statistics were reported as proportions and median values. The $\chi^{2}$ test was used to evaluate the association of tumour characteristics (categorical variables) between NEDP and BMP. The relationship between healthy 
Table V. Sensitivity and specificity of bone turnover and cancer markers (BMP vs. NEDP).

\begin{tabular}{lccc}
\hline Marker & $\%$ AUC $(95 \%$ CI $)$ & $\%$ Sensitivity $(95 \%$ CI $)$ & $\%$ Specificity (95\% CI) \\
\hline CEA & $91.5(85.4-97.6)$ & $48.9(33.7-64.2)$ & $97.1(85.1-99.3)$ \\
CA15-3 & $88.6(81.0-96.2)$ & $64.4(48.8-78.1)$ & $94.4(80.0-100.0)$ \\
OPG & $82.5(74.5-90.6)$ & $74.1(60.4-85.0)$ & $87.7(75.7-94.2)$ \\
OPG + CEA & $93.8(88.7-98.9)$ & $84.4(70.5-93.5)$ & $79.5(63.1-89.6)$ \\
OPG + CA15-3 & $92.2(86.0-98.3)$ & $86.7(73.2-94.9)$ & $72.9(56.8-84.6)$ \\
RANK-L & $69.2(59.0-79.4)$ & $57.4(43.2-70.8)$ & $67.4(53.3-78.7)$ \\
RANK-L+ CEA & $90.7(84.1-97.2)$ & $73.3(58.1-85.4)$ & $50.0(33.9-66.1)$ \\
RANK-L + CA15-3 & $89.4(81.9-96.9)$ & $75.6(60.5-87.1)$ & $47.2(31.9-63.1)$ \\
RANK-L/OPG & $70.0(60.0-80.0)$ & $40.7(27.6-54.9)$ & $77.5(64.0-86.9)$ \\
\hline
\end{tabular}

Cut-off values: CEA, 5 ng/ml; CA15-3, 33 U/ml; OPG, 0.9; RANK-L, 0.6; RANK-L/OPG, 1.0.

individual and patient status and markers was analyzed using non-parametric ranking statistics (Median test). In the absence of internationally accepted cut-off values for OPG and RANK-L markers, the cut-off maximally discriminating between control groups and BMP was identified using receiver operating characteristic (ROC) curve analysis. Ninety-five percent confidence intervals (CI) were calculated for sensitivity and specificity values. Statistical analyses were carried out with SAS Statistical software (version 9.1, SAS Institute, Cary, NC, USA).

\section{Results}

Bone metastasis assessment. The first instrumental diagnostic exam carried out in BMP was scintigraphy (54 patients), PET (5 patients), CT scan (3 patients), and traditional radiography (1 patient). Scintigraphy-based diagnosis was confirmed by CT scan (29 patients), PET (7 patients), MRI (8 patients) biopsy (4 patients) and traditional radiography (4 patients). PET-based diagnosis was confirmed by CT scan (3 patients), biopsy (1 patient), and scintigraphy (1 patient). In the 3 patients first submitted to CT scan, confirmation of bone metastases was made by scintigraphy and also by biopsy in 1 patient. X-raybased diagnosis in 1 patient was confirmed by scintigraphy. All BMP were positive by scintigraphy, but not all lesions were detected, indicating a test sensitivity of $70.7 \%$. In 12 patients $(29.3 \%)$, the remaining lesions were subsequently revealed by CT, PET or MRI.

Biological data. Morphologic, proliferative and hormonal characteristics of primary breast cancers in the two subgroups are shown in Tables I and II. NEDP and BMP differed significantly in terms of tumour grade $(p=0.032$ and $<0.001$, respectively) and proliferation rate, determined by Ki67, and stage ( $p<0.001$ for both). Conversely, the frequency of expressed or amplified HER-2 and ER were equally distributed in the two groups.

With regard to all the circulating markers, median values were independent of age and menopausal status in all subgroups and, in patients with bone metastases, were not correlated with number and type of lesion (lytic, osteoblastic or mixed) or the presence of visceral metastases (Table III). Interestingly, among BMP, the percentage of positivity of RANKL/OPG was higher in patients with lytic lesions, as compared to those with osteoblastic/mixed lesions.

OPG median values were about 3-fold higher in HD (1.9, range 0.6-4.7) and NEDP (1.7, range 0.4-8.9) than in BMP (0.5, range 0.1-5.7) ( $\mathrm{p}<0.001$ for both). Similarly, median RANK-L values were significantly lower in BMP (median value 0.5 , range $0.1-4.5)$ than in $\operatorname{NEDP}(0.8$, range $0.2-3.8 ; \mathrm{p}=0.024)$ or in HD (1.1, range $0.3-3.1 ; \mathrm{p}<0.001)$. RANK-L/OPG was also evaluated as a single marker, with median values of 0.4 (range 0.2-2.1) in HD, 0.5 (range 0.1-2.7) in NEDP and 0.8 (range 0.1-31.2) in BMP (HD vs. BMP: $p=N S$; NEDP vs. BMP: $\mathrm{p}=0.008)$.

CEA and CA15-3 were significantly higher in patients who relapsed in bone sites (4.7, range 0.0-90.6 and 64.8, range 7.1-1538.8, respectively) than in NEDP (1.3, range 0.0-8.3 and 14.1 , range $0.0-36.7$, respectively) ( $\mathrm{p}<0.001$ for both).

Diagnostic relevance. The diagnostic accuracy of single or combined circulating markers was evaluated using continuous values in ROC curve analysis and considering NED patients as the reference group. The area under the ROC curve (AUC) for OPG was 82.5\% (95\% CI, 74.5-90.6) (Table V). OPG, RANK-L, CEA and CA15.3 were not related to each other, and were therefore considered in combination. When OPG was analyzed together with either CEA or CA15-3, the AUC increased to 93.8\% (95\% CI, 88.7-98.9) and 92.2\% (95\% CI, 86.0-98.3), respectively. The AUC value for RANK-L and RANK-L/OPG was lower than that of other markers (Table V) and similarly, the AUC increased when RANK-L was used in combination with CEA or CA15-3.

Sensitivity and specificity were calculated for different cutoff values using the standard $5 \mathrm{ng} / \mathrm{ml}$ for CEA, and $33 \mathrm{U} / \mathrm{ml}$ for CA15-3. Cut-off values for OPG and RANK-L were chosen according to the ROC curves (Table V). An analysis of the diagnostic accuracy of single markers showed a maximum sensitivity for OPG (74.1\%-95\% CI, 60.4-85.0). Sensitivity further increased when OPG was considered in combination with CEA (84.4\%-95\% CI, 70.5-93.5) and even more so when evaluated with CA15-3 (86.7\%-95\% CI, 73.2-94.9). 
RANK-L expression reached 57.4\% (95\% CI, 43.2-70.8) sensitivity and $67.4 \%$ (95\% CI, 53.3-78.7) specificity. Considering RANK-L/OPG as a marker, accuracy was lower than OPG alone (Table V). No relationship between OPG and stage at diagnosis, grading, HER-2, hormonal status, or Ki67 was observed. Furthermore, the association of these markers with OPG did not increase its diagnostic accuracy (data not shown).

Finally, we tested another small negative control group of VM patients to determine if OPG and RANK-L were bone metastases specific. We analyzed 10 patients (4 with liver, 3 with lung, 2 with brain, and 1 with kidney lesions) and results showed a $90 \%$ specificity for OPG and RANK-L, and 70\% for RANK-L/OPG, while both CEA and CA15-3 reached 50\% specificity.

\section{Discussion}

The early diagnosis of bone metastases could be instrumental in bringing forward treatments designed to prevent bone destruction and further serious complications, improving quality of life and increasing overall survival.

In this study, we evaluated an inxpensive, non-invasive test to improve bone metastases diagnosis in patients with breast cancer. To our knowledge, this is the first study to test OPG and RANK-L in the peripheral blood of patients with breast cancer using quantitative RT-PCR. Firstly, we ensured that the two transcripts were not expressed at significantly different levels in pre- and post-menopausal women, both in HD and in each of the patient subgroups. This was an important point to consider before results evaluation, due to the correlation between osteoporosis and menopausal status, and the potential for these markers to be modulated in older donors or patients.

OPG values were found to be about 3 -fold higher in healthy individuals and in disease-free breast cancer patients than in breast cancer patients with bone metastases, independently of the type or extension of bone lesions and the presence or absence of visceral metastases. This observation is supported by the biological rationale that bone metastases from breast cancer are more frequently osteolytic and, thus, are characterized by an increase in bone resorption. A high level of OPG expression could be protective for the development of bone metastases, inhibiting bone resorption through competition for RANK-L with RANK. Due to its low sensitivity, RANK-L does not seem to be suitable for the diagnosis of bone metastases. We compared these markers with those recommended in clinical practice during follow-up visits, namely CEA and CA15-3. We chose these markers, and not other bone specific ones, because our first aim was to understand if OPG and RANK-L could improve upon the results obtained in clinical practice. Furthermore, we decided not to test other bone markers such as NTX, since recent data have shown that NTX levels are not bone metastases specific, and are similar in osteoporotic NEDP and $\operatorname{BMP}(19,20)$. OPG sensitivity was found to be higher than that of the routine markers, and further increased when evaluated in combination with either CEA or CA15-3. We also tested a small series of VM patients, observing OPG and RANK-L expression at very low levels. These preliminary data indicate that these markers, different from CEA and CA15-3, seem to be specific for bone metastases.
At present, the clinical diagnosis of bone metastases is usually made by imaging techniques, such as radiography or Technetium-99 scintigraphy, although it must be underlined that these tests have limitations, especially with regard to early diagnosis and follow-up. Specifically, a radiographic-based diagnosis requires there to be $50 \%$ bone destruction. Scintigraphy has higher sensitivity but lower specificity, especially in pure lytic lesions, because it only detects bone metabolism, thus another imaging study might be needed for an accurate diagnosis (21). It is also an expensive procedure to perform and is neither capable of detecting small bone variations during disease progression nor of predicting response to therapy (21). Although $100 \%$ sensitivity in detecting bone localization has been reported for scintigraphy in the literature, in our experience this value is closer to $70 \%$. Furthermore, many other cases have been diagnosed by other imaging techniques such as CT. Patients operated on for breast cancer currently undergo clinical follow-up with basic blood tests and analysis of circulating tumour markers. In general, instrumental exams are only requested in the presence of symptoms, i.e., scintigraphy for bone pain when bone metastases is already well-established, and clearly, the identification of new, more sensitive markers is of great importance (22).

Currently, there is no single test for the diagnosis of bone metastases that is simple to perform, inexpensive, safe, noninvasive and highly sensitive $(23,24)$. Among the numerous serum markers that have been investigated as potential predictors of clinical outcome in breast cancer patients, the most widely used are CA $15-3$ and CEA $(25,26)$. Some authors have already reported on RANK-L and OPG. In a study on a large series of prostate cancer patients evaluating the clinical relevance of several bone turnover biomarkers, Jung et al identified OPG as the best marker to detect bone metastasis (17). Mountzios et al reported a severe disruption of the RANK-L/OPG axis in patients with bone metastases from solid tumours, including breast cancer (12). Other bone markers of bone resorption, formation, and osteoclastogenesis have been evaluated for their ability to act as indicators of bone metastasis in patients with lung, breast, and prostate cancer $(27,28)$. In breast cancer patients, although cross-linked COOHterminal telopeptides of type I collagen (ICTP), cross-linked $\mathrm{NH} 2$-terminal telopeptides of type I collagen (NTx), and bonespecific alkaline phosphatase (BSAP) have been reported as indicators of bone metastases, none of these show sufficient sensitivity for the early identification of metastases $(15,29)$, and for this reason, none are currently being used in standard clinical practice.

In summary, our results indicated that OPG, rather than RANK-L, used alone or in combination with traditional serum markers, is highly effective in diagnosing bone metastases in breast cancer patients. Confirmation of such findings in our larger ongoing study could open up interesting possibilities for its use as an alternative to radiographic exams or as an aid to the planning of personalized adjuvant bone-targeted therapy.

\section{Acknowledgements}

We thank Professor Rosella Silvestrini for her invaluable scientific contribution and Gráinne Tierney and Dr Ian Seymour for editing the manuscript. 


\section{References}

1. Mundy GR: Metastasis to bone: causes, consequences and therapeutic opportunities. Nat Rev Cancer 2: 584-593, 2002.

2. Jemal A, Siegel R, Ward E, Murray T, Xu J and Thun MJ: Cancer statistics, 2007. CA Cancer J Clin 57: 43-66, 2007.

3. Coleman RE: Clinical features of metastatic bone disease and risk of skeletal morbidity. Clin Cancer Res 12: 6243S-6249S, 2006.

4. Lipton A, Cook R, Saad F, et al: Normalization of bone markers is associated with improved survival in patients with bone metastases from solid tumors and elevated bone resorption receiving zoledronic acid. Cancer 113: 193-201, 2008.

5. Doggrell SA: Clinical efficacy and safety of zoledronic acid in prostate and breast cancer. Expert Rev Anticancer Ther 9: 1211-1218, 2009.

6. Stopeck A, Body JJ, Fujiwara Y, et al: Denosumab versus zoledronic acid for the treatment of breast cancer patients with bone metastases: results of a randomized phase 3 study. Eur J Cancer 7 (Suppl): 2, 2009.

7. Hsu H, Lacey DL, Dunstan CR, et al: Tumor necrosis factor receptor family member RANK mediates osteoclast differentiation and activation induced by osteoprotegerin ligand. Proc Natl Acad Sci USA 96: 3540-3545, 1999.

8. Hofbauer LC, Neubauer A and Heufelder AE: Receptor activator of nuclear factor-kappaB ligand and osteoprotegerin: potential implications for the pathogenesis and treatment of malignant bone diseases. Cancer 92: 460-470, 2001.

9. Blair JM, Zhou H, Seibel MJ and Dunstan CR: Mechanisms of disease: roles of OPG, RANKL and RANK in the pathophysiology of skeletal metastasis. Nat Clin Pract Oncol 3: 41-49, 2006.

10. Mori K, Ando K, Heymann D and Redini F: Receptor activator of nuclear factor-kappa B ligand (RANKL) stimulates boneassociated tumors through functional RANK expressed on bone-associated cancer cells? Histol Histopathol 24: 235-242, 2009.

11. Chen G, Sircar K, Aprikian A, Potti A, Goltzman D and Rabbani SA: Expression of RANKL/RANK/OPG in primary and metastatic human prostate cancer as markers of disease stage and functional regulation. Cancer 107: 289-298, 2006.

12. Mountzios G, Dimopoulos MA, Bamias A, et al: Abnormal bone remodeling process is due to an imbalance in the receptor activator of nuclearfactor-kappaB ligand (RANKL)/osteoprotegerin (OPG) axis in patients with solid tumors metastatic to the skeleton. Acta Oncol 46: 221-229, 2007

13. Coleman R, Brown J, Terpos E, et al: Bone markers and their prognostic value in metastatic bone disease: clinical evidence and future directions. Cancer Treat Rev 34: 629-639, 2008.

14. Coleman RE: The role of bone markers in metastatic bone disease Cancer Treat Rev 32 (Suppl 1): 1-2, 2006.

15. Garnero P, Buchs N, Zekri J, Rizzoli R, Coleman RE and Delmas PD: Markers of bone turnover for the management of patients with bone metastases from prostate cancer. Br J Cancer 82: 858-864, 2000.
16. Ulrich U, Rhiem K, Schmolling J, et al: Cross-linked type I collagen $\mathrm{C}$ - and $\mathrm{N}$-telopeptides in women with bone metastases from breast cancer. Arch Gynecol Obstet 264: 186-190, 2001.

17. Jung K, Lein M, Stephan C, et al: Comparison of 10 serum bone turnover markers in prostate carcinoma patients with bone metastatic spread: diagnostic and prognostic implications. Int J Cancer 111: 783-791, 2004.

18. Ricotti L, Barzanti F, Tesei A, et al: Combined 4-hydroxyifosfamide and vinorelbine treatment in established and primary human breast cell cultures. Ann Oncol 11: 587-594, 2000.

19. Gonnelli S, Cadirni A, Caffarelli C, et al: Changes in bone turnover and in bone mass in women with breast cancer switched from tamoxifen to exemestane. Bone 40: 205-210, 2007.

20. Coleman RE, Major P, Lipton A, et al: Predictive value of bone resorption and formation markers in cancer patients with bone metastases receiving the bisphosphonate zoledronic acid. J Clin Oncol 23: 4925-4935, 2005.

21. Costelloe CM, Rohren EM, Madewell JE, et al: Imaging bone metastases in breast cancer: techniques and recommendations for diagnosis. Lancet Oncol 10: 606-614, 2009.

22. Pestalozzi B and Castiglione M: Primary breast cancer: ESMO clinical recommendations for diagnosis, treatment and follow-up. Ann Oncol 19 (Suppl 2): 7-10, 2008

23. Ben-Haim S and Israel O: Breast cancer: role of SPECT and PET in imaging bone metastases. Semin Nucl Med 39: 408-415, 2009.

24. Ebert W, Muley T, Herb KP and Schmidt-Gayk H: Comparison of bone scintigraphy with bone markers in the diagnosis of bone metastasis in lung carcinoma patients. Anticancer Res 24: 3193-3201, 2004

25. Nicolini A, Tartarelli G, Carpi A, et al: Intensive post-operative follow-up of breast cancer patients with tumour markers: CEA TPA or CA15.3 vs MCA and MCA-CA15.3 vs CEA-TPA-CA15.3 panel in the early detection of distant metastases. BMC Cancer 6: 269,2006

26. Nicolini A, Carpi A and Tarro G: Biomolecular markers of breast cancer. Front Biosci 11: 1818-1843, 2006.

27. Clamp A, Danson S, Nguyen H, Cole D and Clemons M: Assessment of therapeutic response in patients with metastatic bone disease. Lancet Oncol 5: 607-616, 2004

28. Costa L, Demers LM, Gouveia-Oliveira A, et al: Prospective evaluation of the peptide-bound collagen type I cross-links $\mathrm{N}$-telopeptide and $\mathrm{C}$-telopeptide in predicting bone metastases status. J Clin Oncol 20: 850-856, 2002.

29. Kiuchi K, Ishikawa T, Hamaguchi Y, et al: Cross-linked collagen $\mathrm{C}$ - and $\mathrm{N}$-telopeptides for an early diagnosis of bone metastasis from breast cancer. Oncol Rep 9: 595-598, 2002. 